\title{
利用光子晶体抑制大气窗口发射辐射的 优化设计及实验研究
}

\author{
李进, 叶宏* \\ 中国科学技术大学热科学与能源工程系, 合肥 230026 \\ *E-mail: hye@ustc.edu.cn
}

收稿日期: 2009-07-30; 接受日期: 2009-10-28

国家重点基础研究发展计划(“973 计划”)(批准号: 2009CB939900)资助项目

\begin{abstract}
摘要利用传输矩阵法讨论了光子晶体对大气窗口 $(8 \sim 14 \mu \mathrm{m})$ 内发射辐射的抑制作用. 根据材料的光学特性, 选择了 $\mathrm{Ge}$ 与 $\mathrm{ZnS}$ 作为光子晶体的组成材料. 对光子晶体的结构进 行了优化, 优化值为 $\mathrm{Ge}$ 与 $\mathrm{ZnS}$ 的厚度分别为 0.63 和 $1.11 \mu \mathrm{m}$, 光学厚度比为 $1: 1$, 周期数 为 8 . 利用蒸发镀膜制备了光子晶体, 并对制得的光子晶体的光学性质进行了测定, 验证

关键词

光子晶体

大气窗口发射辐射

抑制 光子晶体对大气窗口发射辐射的抑制作用.
\end{abstract}

光子晶体的概念最先由 Yablonovitch ${ }^{[1]}$ 提出, 其 定义为因介电常数空间周期性分布而具有光子带隙 的特殊材料. 光子晶体的特性在于存在光子禁带, 频 率处于禁带中的电磁波不能在其中传播. 随着纳米 尺度加工技术的发展, 研究人员发现利用光子晶体可 以改变材料固有的宏观辐射特性 ${ }^{[2]}$, 即材料的光谱发 射率在某些特定光谱区域内会出现峰值或被抑制.

通常可以采用平面波法、传输矩阵法、有限时域 差分法等来对光子晶体进行研究 ${ }^{[3]}$. 平面波法用得较 早而且较多, 其思想是根据布洛赫理论, 将光子晶体 的介电常数与电磁场用平面波展开, 求解本征方程, 即得到光子能带, 但是当介电常数随频率变化时, 则 无法求解 ${ }^{[4]}$. 有限时域差分法由 Yee 于 1966 年首次 提出, 对电磁场的 $E$ 和 $H$ 分量采取空间和时间上的交 替抽样的离散方式, 得到含时间变量的差分方程组, 沿时间轴逐步推进可求解空间电磁场, 优点是计算 过程较为直观 ${ }^{[5]}$. 本文采用传输矩阵法 ${ }^{[6]}$ 来讨论光子
晶体对大气窗口 8 14 $\mu \mathrm{m}$ 波段内发射辐射的抑制作 用, 该方法具有矩阵小、阵元少的优点, 计算量较低, 而且针对介电常数随波长变化的系统尤为有效.

本文选择了 $\mathrm{Ge}$ 与 $\mathrm{ZnS}$ 作为光子晶体组成材料, 并分别选择 $\mathrm{SiC}$ 和 $\mathrm{Al}$ 为基底. $\mathrm{Ge}$ 与 $\mathrm{ZnS}$ 均属于红外 波段的透明材料, 其中 $\mathrm{Ge}$ 的折射率较高, $\mathrm{ZnS}$ 的较低 为了较好地抑制 $8 \sim 14 \mu \mathrm{m}$ 内的发射辐射, 根据光子 晶体的禁带特性, 对光子晶体的结构进行了优化设 计,并根据优化设计结果, 采用蒸发镀膜方法制备了 光子晶体, 测试了其光学特性, 较为系统地验证了在 大气窗口 $8 \sim 14 \mu \mathrm{m}$ 波段内光子晶体对表面发射辐射 的抑制作用.

\section{1 理论分析}

\section{1 数学模型}

图 1 为一维光子晶体结构示意图, 介质 1 和 2 的

引用格式: Li J, Ye H. Optimization of the inhibition of atmospheric window emission using photonic crystals. Sci China Tech Sci, 2010, 53: 1315-1319, doi: 10.1007/s11431-010-0136-y 


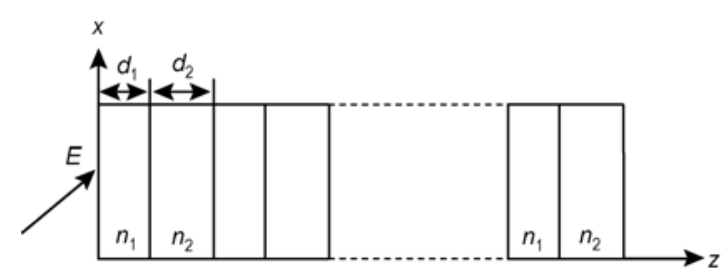

图 1 一维光子晶体结构示意图

折射率分别为 $n_{1}$ 和 $n_{2}$, 厚度分别为 $d_{1}$ 和 $d_{2}$, 它们在 某一方向上周期性排列构成光子晶体, 最小的周期 单元称为晶胞单元, 厚度为 $d_{1}+d_{2}$.

设电磁波角频率为 $\omega$, 则在光子晶体中传播时, 其电矢量 $\boldsymbol{E}(x, y, z, t)$ 满足下式 ${ }^{[6]}$

$$
\boldsymbol{E}(x, y, z, t)=u(z) \mathrm{e}^{\mathrm{i} K z} \mathrm{e}^{\mathrm{i}\left(k_{x} x+k_{y} y-\omega t\right)},
$$

上式中, $k_{x}$ 和 $k_{y}$ 是波矢的分量, $K$ 是布洛赫波数.

选取光子晶体中任意第 $v$ 层介质膜作研究对象, 如图 2 所示. 用下标 $\mathrm{R}$ 表示右行波, 下标 $\mathrm{L}$ 表示左行 波, 则 $E_{\mathrm{V}}$ 和 $E_{\mathrm{V}}$ 分别表示第 $v$ 层界面左侧左行波和右 行波的电分量, $\tilde{E}_{v \mathrm{~L}}$ 和 $\tilde{E}_{v \mathrm{R}}$ 表示左行波和右行波的复 振幅. $E_{v \mathrm{~L}}^{\prime}$ 和 $E_{v \mathrm{R}}^{\prime}$ 分别表示第 $v$ 层界面右侧左行波和 右行波的电分量, $\tilde{E}_{v \mathrm{~L}}^{\prime}$ 和 $\tilde{E}_{v \mathrm{R}}^{\prime}$ 表示左行波和右行波的 复振幅. 对磁分量也可采用同样的命名方式.

反射系数 $r$ 定义为反射波(左行波)和入射波(右 波)的振幅之比，即

$$
r=\frac{E_{v \mathrm{~L}}}{E_{v \mathrm{R}}}
$$

在图 2 中, 对于第 $v$ 层界面, 利用电磁场的连续 条件可得

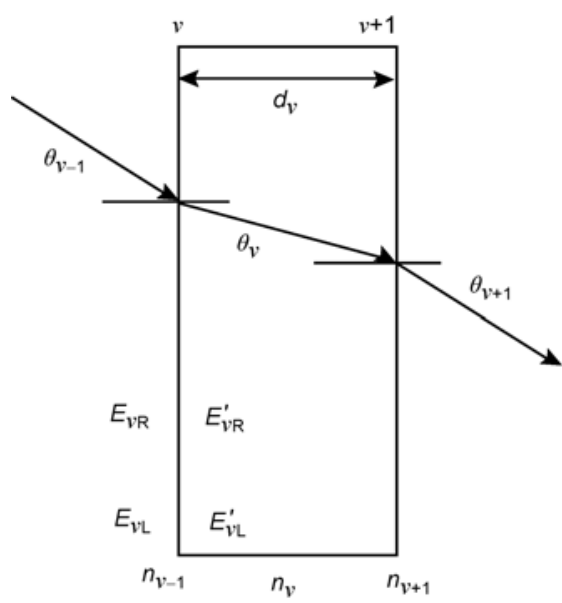

图 2 第 $v$ 层介质膜光路示意图

$$
\begin{aligned}
& E_{v \mathrm{~L}}+E_{v \mathrm{R}}=E_{v \mathrm{~L}}^{\prime}+E_{v \mathrm{R}}^{\prime}, \\
& \eta_{v-1} E_{v \mathrm{R}}-\eta_{v-1} E_{v \mathrm{~L}}=\eta_{v}\left(E_{v \mathrm{R}}^{\prime}-E_{v \mathrm{~L}}^{\prime}\right),
\end{aligned}
$$

上式中, $\eta_{v}$ 和 $\eta_{v-1}$ 分别是第 $v$ 层介质和第 $v-1$ 层介质的 有效光学导纳.

引入光纳矩阵:

$$
\boldsymbol{V}_{v}=\left(\begin{array}{cc}
1 & 1 \\
\eta_{v} & -\eta_{v}
\end{array}\right), \quad \boldsymbol{V}_{v}^{-1}=\left(\begin{array}{cc}
1 & \eta_{v}^{-1} \\
1 & -\eta_{v}^{-1}
\end{array}\right) .
$$

电磁波在第 $v$ 层介质膜中传播时, 位相变化为

$$
\left(\begin{array}{c}
E_{v \mathrm{R}}^{\prime} \\
E_{v \mathrm{~L}}^{\prime}
\end{array}\right)=\left(\begin{array}{ll}
\mathrm{e}_{v}^{\mathrm{i} \varphi_{v}} & 0 \\
0 & \mathrm{e}_{v}^{-\mathrm{i} \varphi_{v}}
\end{array}\right)\left(\begin{array}{l}
E_{v+1, \mathrm{R}} \\
E_{v+1, \mathrm{~L}}
\end{array}\right),
$$

其中, $\varphi_{v}=\frac{2 \pi}{\lambda} n_{v} d_{v} \cos \theta_{v}$ 是光在第 $v$ 层膜中穿行一次 的相移.

根据薄膜光学理论 ${ }^{[7]}$, 电磁波在每层介质中的传 输特性可用一个 $2 \times 2$ 的特征矩阵来表示. 对于第 $v$ 层 介质, 其特征矩阵为

$$
\boldsymbol{M}_{v}=\left(\begin{array}{ll}
\cos \varphi_{v} & \mathrm{i} \sin \varphi_{v} / \eta_{v} \\
\mathrm{i} \eta_{v} \sin \varphi_{v} & \cos \varphi_{v}
\end{array}\right),
$$

上式中, $\varphi_{v}=\frac{2 \pi}{\lambda} n_{v} d_{v} \cos \theta_{v}, n_{v}$ 和 $d_{v}$ 分别是第 $v$ 层介质 的折射率及厚度， $\lambda$ 为参考波长， $\theta_{v}$ 是电磁波的传播 方向与界面法线方向之间的夹角. $\eta_{v}$ 是介质的有效光 学导纳, 表达式为 $\eta_{v}=H_{\mathrm{t}} / E_{\mathrm{t}}, H_{\mathrm{t}}$ 表示磁分量在介质 交界面的切向分量, $E_{\mathrm{t}}$ 表示电分量在介质交界面的切 向分量.

对于由折射率分别为 $n_{1}$ 和 $n_{2}$ 的 2 种介质组成的 一个基本周期单元来说, 特征矩阵如下

$$
\boldsymbol{M}_{0}=\left(\begin{array}{cc}
\cos \varphi_{1} & \mathrm{i} \sin \varphi_{1} / \eta_{1} \\
\mathrm{i} \eta_{1} \sin \varphi_{1} & \cos \varphi_{1}
\end{array}\right)\left(\begin{array}{cc}
\cos \varphi_{2} & \mathrm{i} \sin \varphi_{2} / \eta_{2} \\
\mathrm{i} \eta_{2} \sin \varphi_{2} & \cos \varphi_{2}
\end{array}\right) .
$$

如果一维光子晶体是由 $s$ 个这样的基本周期组 成, 则光子晶体的总特征矩阵为 $s$ 个 $\boldsymbol{M}_{0}$ 的乘积, 表示为

$$
\boldsymbol{M}=\boldsymbol{M}_{0}^{s}=\left(\begin{array}{ll}
m_{11} & m_{12} \\
m_{21} & m_{22}
\end{array}\right),
$$

上式中, $m_{11}, m_{12}, m_{21}$ 和 $m_{22}$ 是 $\boldsymbol{M}$ 的矩阵元.

当在基底上加入光子晶体后, 反射系数的表达 式如下 ${ }^{[7]}$

$$
r_{\lambda, \theta}=\frac{\left(m_{11}+m_{12} \cdot \eta_{g}\right) \cdot \eta_{o}-\left(m_{22} \cdot \eta_{g}+m_{21}\right)}{\left(m_{11}+m_{12} \cdot \eta_{g}\right) \cdot \eta_{o}+\left(m_{22} \cdot \eta_{g}+m_{21}\right)},
$$


其中, $\eta_{o}$ 为空气的有效光学导纳, $\eta_{g}$ 为基底的有效光 学导纳.

从而得到反射率 $R_{\lambda, \theta}$ 如下

$$
R_{\lambda, \theta}=\left|r_{\lambda, \theta}\right|^{2} .
$$

给定边界条件透射率 $\tau=0$, 可得 $\alpha_{\lambda, \theta}+R_{\lambda, \theta}=1$, 根据基尔霍夫定律 ${ }^{[8]}$ 可得到

$$
\varepsilon_{\lambda, \theta}=\alpha_{\lambda, \theta}=1-R_{\lambda, \theta} .
$$

将(10)式中的 $R_{\lambda, \theta}$ 代入上式, 并通过对角度进行 积分, 相应地可以得到其半球向发射率随波长的变化.

\section{2 结果与分析}

本文采用的光子晶体的结构为 $\mathrm{A}\left|(\mathrm{LH})^{s}\right| \mathrm{G}, \mathrm{A}$ 表示 空气, $\mathrm{G}$ 表示基底, $s$ 表示周期数. $\mathrm{L}$ 与 $\mathrm{H}$ 分别表示低 折射率介质 $\mathrm{ZnS}$ 与高折射率介质 $\mathrm{Ge}$. 针对大气窗口 $8 \sim 14 \mu \mathrm{m}$ 波段表面的发射辐射, 对光子晶体进行了优 化设计.

根据优化值, 采用蒸发镀膜的方法在 $\mathrm{SiC}$ 和 $\mathrm{Al}$ 基底上制备了 $\mathrm{Ge} / \mathrm{ZnS}$ 型光子晶体, 通过对制得样品 的光谱半球向反射率进行测定, 将理论值与实际值 对比, 以此来讨论光子晶体对材料表面发射辐射的 抑制作用.

\section{1 高低折射率介质的光学厚度比的影响}

根据薄膜光学理论可知 ${ }^{[7]}$, 当一个周期内高低折 射率介质的光学厚度之和为 $1 / 2$ 波长的时候, 存在反 射带. 当高低折射率介质的厚度均为 $1 / 4$ 波长时(即等 厚膜系), 主反射带的宽度最大, 可较大程度上满足 工作的目的. 表 1 表示的是不同周期数下, 在 $8 \sim 14$ $\mu \mathrm{m}$ 内 $100^{\circ} \mathrm{C}$ 的 $\mathrm{SiC}$ 基底的总发射功率随高低折射率 介质厚度比的变化, 对比分析可得, 当高低折射率介 质的光学厚度比为 $1: 1$ 时, 其发射功率最小, 此时 光子晶体形成的反射带最大.

表 1 不同周期数下, $100^{\circ} \mathrm{C}$ 的 $\mathrm{SiC}$ 基底在大气窗口波段内 $(8 \sim 14 \mu \mathrm{m})$ 的总发射功率随高低折射率介质光学厚度比的 变化 $\left(\mathbf{W} / \mathbf{m}^{2}\right)$

\begin{tabular}{cccccccc}
\hline & $1 / 4: 7 / 4$ & $2 / 4: 3 / 4$ & $3 / 4: 5 / 4$ & $1: 1$ & $5 / 4: 3 / 4$ & $3 / 4: 2 / 4$ & $7 / 4: 1 / 4$ \\
\hline$s=2$ & 68.47 & 55.55 & 50.85 & 49.96 & 58.12 & 68.62 & 70.81 \\
$s=4$ & 57.46 & 37.75 & 27.97 & 27.00 & 38.04 & 55.72 & 69.96 \\
$s=6$ & 54.10 & 35.24 & 24.74 & 24.32 & 33.90 & 52.99 & 69.93 \\
$s=8$ & 51.13 & 33.14 & 24.21 & 23.83 & 33.70 & 52.09 & 67.12 \\
\hline
\end{tabular}

\section{2 周期数 $s$ 的影响}

图 3 表示的是当光子晶体的禁带中心波长取为 $11 \mu \mathrm{m}$ 时, 以 $\mathrm{SiC}$ 为基底的光谱半球向发射率随波长 的变化. 可以看出, 当周期数 $s$ 从 2 开始变化, 随着 周期数的增加, 对表面的发射辐射的抑制作用增强, 当周期数增加到 8 时, 即使再改变周期数, 抑制效果 变化很小. 所以, 周期数为 8 是较为合理的选择.

图 4 表示的是基底为 $\mathrm{Al}$ 时的光谱半球向发射率 随波长的变化. 可以看出, 随着周期数的增加, 在 $8 \sim 14 \mu \mathrm{m}$ 内其发射率均较低, 发射辐射得到了较好的 抑制. 当周期数为 8 时, 已达到了较好的抑制作用. 即使周期数再增加, 抑制效果变化不明显, 其光谱半 球向发射率变化不大.

\section{3 禁带中心波长的影响}

选取 $\mathrm{Ge} / \mathrm{ZnS}$ 型光子晶体的周期数为 8 , 高低折

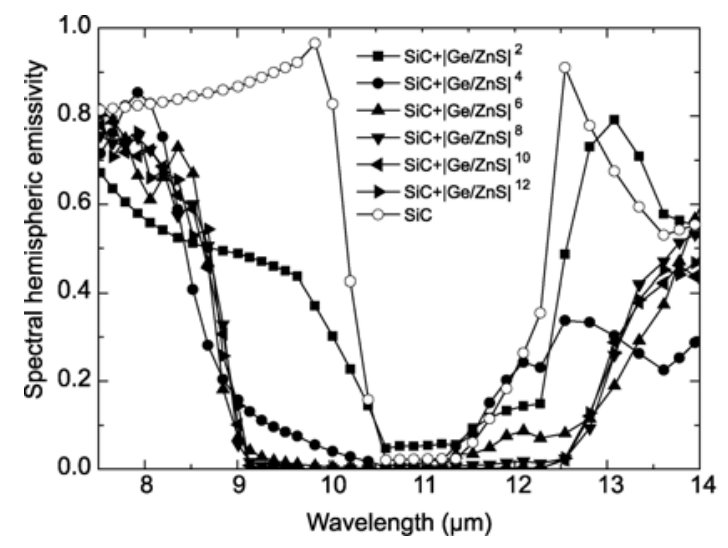

图 $3 \mathrm{SiC}$ 为基底时, 光谱半球向发射率随波长的变化

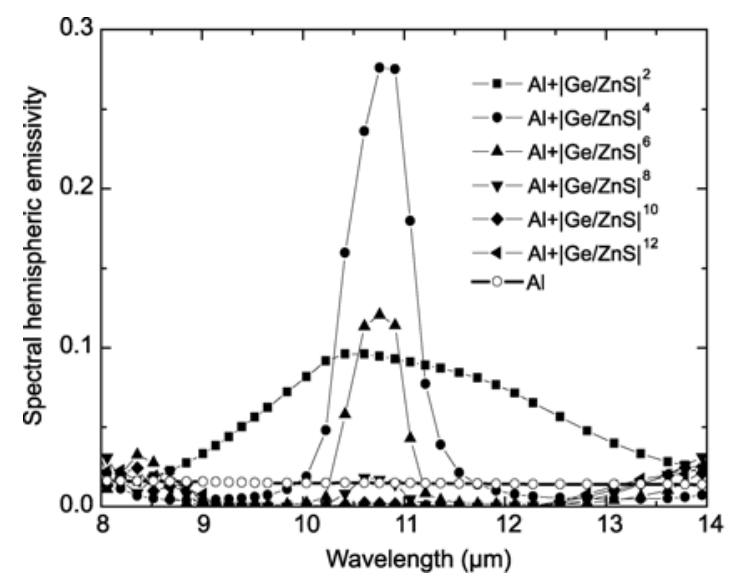

图 $4 \mathrm{Al}$ 为基底时, 光谱半球向发射率随波长的变化 
射率介质的光学厚度比为 $1: 1$. 考虑到需要抑制的波 段是 $8 \sim 14 \mu \mathrm{m}$, 选择不同的禁带中心波长进行计算比 较, 拟得到最佳的禁带中心波长.

由图 5 可得, 基底为 $\mathrm{SiC}$, 禁带中心波长为 10.0 $\mu \mathrm{m}$ 时, 此时组合表面的发射功率最小. 加入 $\mathrm{Ge} / \mathrm{ZnS}$ 光子晶体后, 可使 $\mathrm{SiC}$ 的发射功率从 78.84 降至 15.42 $\mathrm{W} / \mathrm{m}^{2}$. 当基底为 $\mathrm{Al}$ 时, 禁带中心波长从 $10.0 \mu \mathrm{m}$ 变 化到 $11.0 \mu \mathrm{m}$ 时, 组合表面的发射功率均较低, 加入 $\mathrm{Ge} / \mathrm{ZnS}$ 光子晶体后, 可使 $\mathrm{Al}$ 的发射功率从 2.05 降至 $1.03 \mathrm{~W} / \mathrm{m}^{2}$. 根据四分之一波长理论 ${ }^{[7]}$, 禁带中心波长 取为 $10.0 \mu \mathrm{m}$ 时, 相应地 $\mathrm{Ge}$ 和 $\mathrm{ZnS}$ 薄膜的厚度分别 为 0.63 和 $1.11 \mu \mathrm{m}$. 但是由于加工工艺的问题, 薄膜 厚度需做至 $0.70 \mu \mathrm{m}$ 以上, 所以取光子晶体的禁带中 心波长为 $11.0 \mu \mathrm{m}$, 相应地 $\mathrm{Ge}$ 及 $\mathrm{ZnS}$ 薄膜的厚度分 别为 0.70 和 $1.22 \mu \mathrm{m}$.

\section{4 制备样品及对比分析}

以 $\mathrm{SiC}$ 及 $\mathrm{Al}$ 为基底, 采用蒸发镀膜的方法制备 光子晶体. 光子晶体的结构为 $A\left|(Z n S G e)^{8}\right| G, G$ 为基 底, $\mathrm{A}$ 为空气, 按照交替结构的次序, 依次镀上 $\mathrm{Ge}$ 和 $\mathrm{ZnS}$, 共 8 个周期. 通过对制得样品的光谱半球向反 射率进行测定, 由于基底不透明, 透射率为零, 根据 基尔霍夫定律可以得出其光谱半球向发射率曲线.

图 6 给出的是 $\mathrm{SiC}$ 与 $\mathrm{Al}$ 为基底时, 加入光子晶 体后, 测定的光谱半球向反射率随波长的变化. 所采 用的仪器是 Bruke 公司的 EQUINOX55 型傅立叶变换 红外光谱仪 (积分球附件为 A562). 对于测试样品来 说, 若其镜反射量比较大, 就会出现反射率在 $100 \%$ 附近的情况. 从图 6 中可以看出, 在大气窗口 $8 \sim 14 \mu \mathrm{m}$

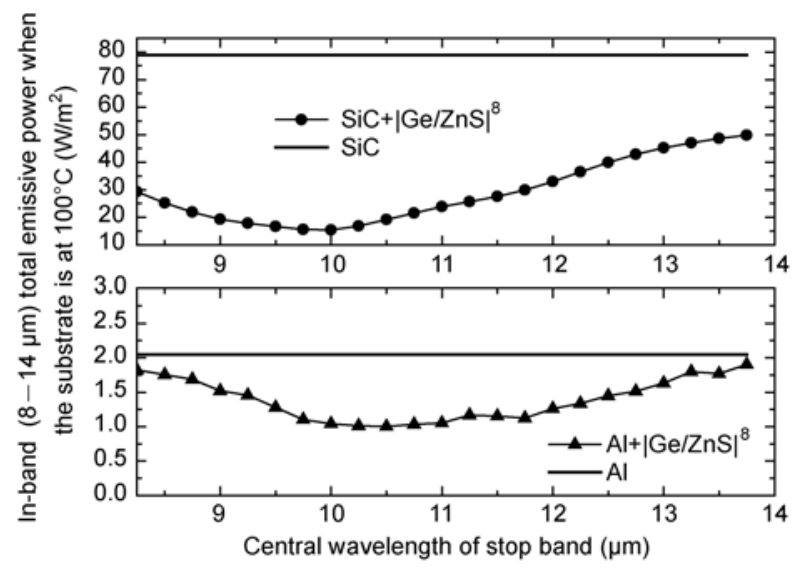

图 5 基底为 $100^{\circ} \mathrm{C}$ 时的总发射功率随禁带中心波长的变化

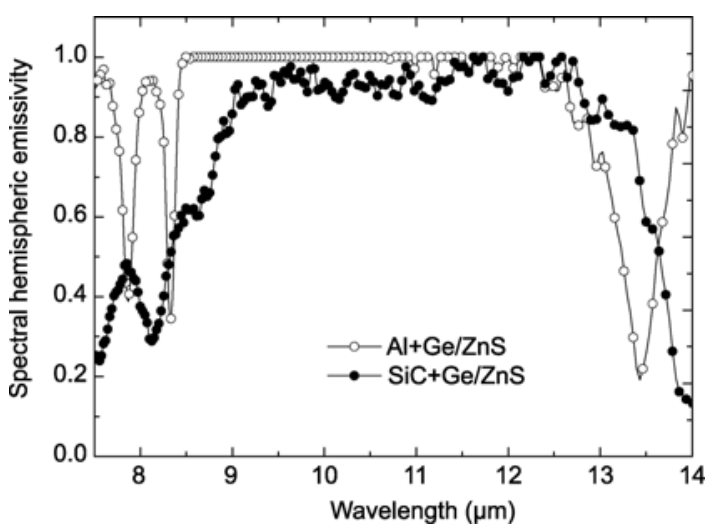

图 $6 \mathrm{Al}$ 与 $\mathrm{SiC}$ 为基底时, 加入光子晶体后的光谱半球 向反射率随波长的变化(测量值)

波段内, 所得 2 种样品的反射率较高, 均超过 $90 \%$. 由于铝表面相对 $\mathrm{SiC}$ 更平整光滑些, 所以反射率相对 地更高一些.

图 7 表示的是 $\mathrm{SiC}$ 为基底时, 光谱半球向发射率 随波长的变化. 比较曲线 (a) 与 (c) 可以看出, 加入光 子晶体对 $\mathrm{SiC}$ 表面在 $8 \sim 14 \mu \mathrm{m}$ 发射辐射起到了较好 的抑制作用, 大部分区域内光谱半球向发射率的值 均较低. 比较曲线(a)与(b)可以看出, $\mathrm{SiC}$ 为基底时, 在 8.7 12.7 $\mu \mathrm{m}$ 波段内, 加入光子晶体后其光谱半球 向发射率的实测值与计算值较为符合.

图 8 表示的是 $\mathrm{Al}$ 为基底时, 光谱半球向发射率 随波长的变化. 比较(a)与(c)可看出, 在 $8.5 \sim 12.5 \mu \mathrm{m}$ 波段内, $\mathrm{Al}$ 表面的发射率得到了较好的抑制. 比较 (a)与(b)可得，该波段处于光子晶体的主禁带，实验 值与计算值符合得比较好. 在 $8 \sim 14 \mu \mathrm{m}$ 的 2 个边缘波

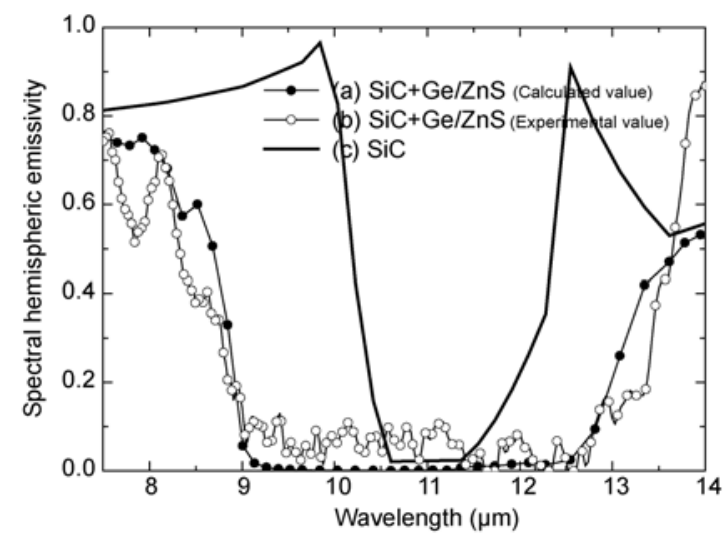

图 $7 \mathrm{SiC}$ 为基底时, 光谱半球向发射率随波长 的变化(结果对比) 


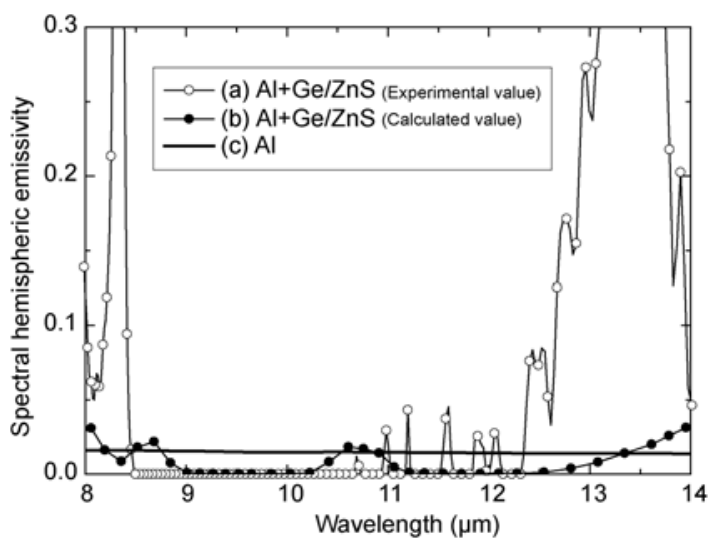

图 $8 \mathrm{Al}$ 为基底时, 光谱半球向发射率随波长 的变化(结果对比)

段处, 存在着 2 个吸收峰, 相应地发射率较高. 对于 光子晶体来讲, 有若干禁带, 其中主禁带最宽, 发射 率最低, 能在最大的光谱区间内抑制发射辐射, 次禁 带次之, 而位于主禁带与次禁带光谱区间内的发射率 较高. 该实验采取的主禁带中心波长为 $11 \mu \mathrm{m}$, 由于 制备的是多层薄膜, 所得光子晶体的主禁带宽度比 计算值略低, 以致于 $8 \sim 8.5$ 与 12.5 14 $\mu \mathrm{m}$ 这 2 个波段
位于光子晶体的主禁带和次禁带之间, 发射率相对 较高.

\section{3 结论}

利用光子晶体的禁带特性来抑制大气窗口 8 14 $\mu \mathrm{m}$ 波段内表面的发射辐射. 根据材料红外特性及物 理性质, 选择了 $\mathrm{Ge}$ 与 $\mathrm{ZnS}$ 作为光子晶体的组成材料. 为较好地抑制大气窗口 $8 \sim 14 \mu \mathrm{m}$ 内的发射辐射, 对 光子晶体的结构进行了优化. 光子晶体结构的优化 值是 $\mathrm{Ge}$ 与 $\mathrm{ZnS}$ 的厚度分别为 0.63 和 $1.11 \mu \mathrm{m}$, 周期 数为 8 , 高低折射率介质的光学厚度比为 $1: 1$. 由于 制作工艺的限制, $\mathrm{Ge}$ 与 $\mathrm{ZnS}$ 的厚度需选为 0.70 和 1.22 $\mu \mathrm{m}$. 根据优化结果, 采用蒸发镀膜的方法制备了光 子晶体. 对样品的光学特性进行测定, 在大气窗口 8 14 $\mu \mathrm{m}$ 内, 加入光子晶体的 $\mathrm{Al}$ 基底的组合表面光 谱半球向反射率可达到 $100 \%, \mathrm{SiC}$ 基底的组合表面的 反射率也较高, 接近 $100 \%$. 理论值与实验值符合较 好, 进而从理论与实验 2 个角度验证了光子晶体对表 面发射辐射的抑制作用.

\section{参考文献}

1 Yablonovitch E. Inhibited spontaneous emission in solid-state physics and electrics. Phys Rev Lett, 1987, 58: 2059-2062

2 Yablonovitch E, Gmitter T J. Photonic band structure: The face-centered-cubic case employing nonspherical atoms. Phys Rev Lett, 1991, 67: $2295-2298$

3 Joannopoulos J D, Johnson S G, Winn J N, et al. Photonic Crystals: Molding the Flow of Light. 2nd ed. Princeton: Princeton University Press, 2008

4 Shi S Y, Chen C H, Prather D W. Revised plane wave method for dispersive material and its application to band structure calculations of photonic crystal slabs. Appl Phys Lett, 2005, 86: 043104

5 Oubre C, Nordlander P. Optical properties of metallodielectric nanostructures calculated using the finite difference time domain method. J Phys Chem B, 2004, 108: 17740-17747

6 Felbacq D, Guizal B, Zolla F. Wave propagation in one-dimensional photonic crystals. Optics Commun, 1998, 152: 119-126

7 唐晋发, 顾培夫, 刘旭, 等. 现代光学薄膜技术. 杭州: 浙江大学出版社, 2007

8 Incropera F P, DeWitt D P, Bergman T L, et al. Fundamentals of Heat and Mass Transfer. 6th ed. Ge X S, Ye H, Translation. Beijing: Chemical Industry Press, 2007

9 Incropera F P, DeWitt D P, Bergman D P, et al. 传热和传质的基本原理. 第六版. 葛新石, 叶宏, 译. 北京：化学工业出版社, 2007 